\title{
Trastornos del sueño- vigilia como marcadores de ideación suicida: una revisión sistemática de la literatura
}

\author{
Sleep disorders - watch as markers of the suicide ideation: a systematic review of literature \\ Distúrbios do sono - vigília como marcadores da ideação suicida: uma revisão sistemática da
}

literatura

Recibido: 06/02/2021 | Revisado: 07/02/2021 | Acepto: 10/02/2021 | Publicado: 18/02/2021

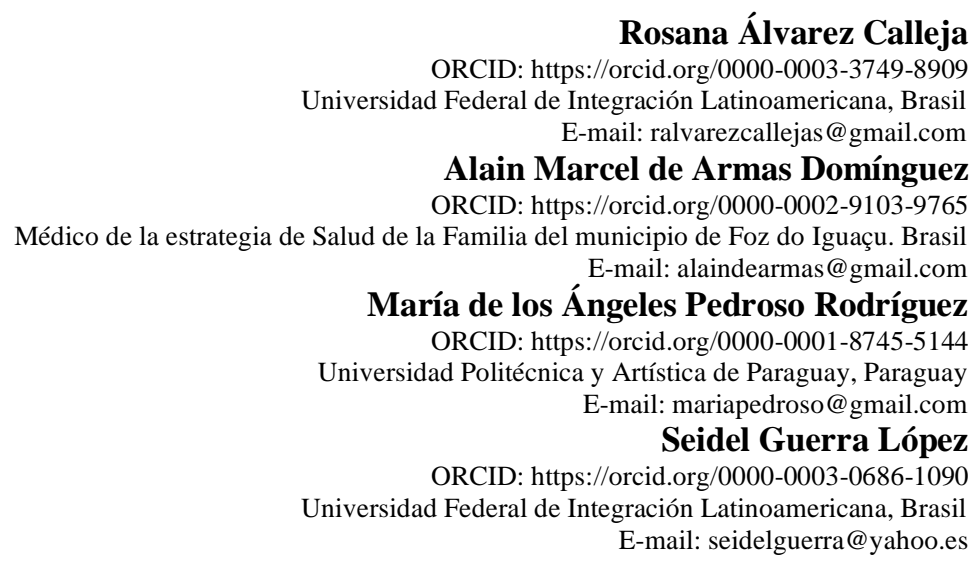

\section{Resumen}

Se realizó una revisión sistemática de la literatura con el objetivo de determinar si los trastornos del ciclo sueño-vigilia pueden ser considerados marcadores de ideación suicida. Para llevar a cabo la revisión y responder la pregunta de investigación fue llevada a cabo una búsqueda en las bases de datos bibliográficos del área de la salud: Medline, Scielo y LILACS durante los meses de mayo a diciembre de 2020. Se incluyeron estudios originales cuantitativos y revisiones cualitativas, así como meta-análisis, publicados en los últimos 10 años en idiomas español, inglés y portugués. Fueron encontrados 39 artículos que cumplieron con los criterios de inclusión, de los cuales 37 estaban escritos en idioma inglés y 2 en idioma portugués. La base de datos Medline aportó el 79,4 \% de los artículos utilizados. El 87, $1 \%$ de los artículos se corresponden con estudios originales cuantitativos. La presenta revisión sistemática evidenció que los trastornos del sueño son un factor de riesgo independiente en relación con la conducta suicida en diferentes contextos clínicos y entornos socio-culturales.

Palabras clave: Trastornos del sueño-vigilia; Depresión; Ideación suicida.

\begin{abstract}
A systematic review of the literature was carried out with the aim of determining whether sleep-wake cycle disorders can be considered markers of suicidal ideation. To carry out the review and answer the research question, a search was conducted in the bibliographic databases of the health area: Medline, Scielo and LILACS during the months of may to december 2020. Original quantitative studies and qualitative reviews were included, as well as meta-analyses, published in the last 10 years in Spanish, English and Portuguese. Thirty-nine articles were found that met the inclusion criteria, of which 37 were written in English and 2 in Portuguese. The Medline database contributed $79.4 \%$ of the articles used. Of the articles, $87.1 \%$ corresponded to original quantitative studies. The present systematic review showed that sleep disorders are an independent risk factor for suicidal behavior in different clinical contexts and socio-cultural settings.
\end{abstract} Keywords: Sleep-wake disorders; Depression; Suicidal ideation.

\section{Resumo}

Foi realizada uma revisão sistemática da literatura com o objetivo de determinar se as perturbações do ciclo sono-vigília podem ser consideradas marcadores de ideação suicida. A fim de realizar a revisão e responder à questão da investigação, foi realizada uma pesquisa nas bases de dados bibliográficas da área da saúde: Medline, Scielo e LILACS durante os meses de maio a dezembro de 2020. Foram incluídos estudos quantitativos originais e revisões qualitativas, bem como meta-análises, publicadas nos últimos 10 anos em espanhol, inglês e português. Foram encontrados trinta e nove artigos que preenchiam os critérios de inclusão, dos quais 37 foram escritos em inglês e 2 em português. A base de dados Medline contribuiu com 79,4\% dos artigos utilizados. Os 87,1 \% dos artigos correspondiam a estudos 
quantitativos originais. A presente revisão sistemática mostrou que as perturbações do sono são um fator de risco independente para o comportamento suicida em diferentes contextos clínicos e cenários socioculturais.

Palavras-chave: Distúrbios sono-vigília; Depressão; Tendência suicida.

\section{Introducción}

Los trastornos del sueño constituyen un problema de salud frecuente en la práctica clínica. Los disturbios del patrón y la periodicidad del sueño REM (Rapid Eyes Movement) y no REM son la base fisiopatológica de estas entidades (Berry et al., 2020) El ciclo sueño vigilia está regulado por complejos procesos biológicos, en los cuales el núcleo supraquiasmático localizado en el hipotálamo desempeña uma importante función. Múltiples neurotransmisores participan del proceso de regulación del ciclo sueño vigilia. Entre ellos podemos citar a la serotonina del núcleo dorsal del rafe, La norepinefrina de las neuronas del locus cerúleo, La acetilcolina de La formación reticular y la dopamina. La disrupción del equilibrio del sistema de mensajeros químicos repercute en los parámetros fisiológicos, biológicos, comportamentales e eletroencefalográfico del sueño REM y no REM (Takahashi, 2017). Los trastornos del ritmo circadiano endógenos se caracterizan por una alineación inadecuada entre el tiempo de sueño y el ambiente físico o social del sujeto. Los trastornos del ritmo circadiano deben ser diferenciados de los trastornos de sueño primarios y secundarios a otras entidades clínicas o a enfermedades psiquiátricas (Kalmbach et al., 2017).

Los trastornos del sueño primarios se clasifican en parasomnias y disomnias. Las parasomnias son experiencias inusuales que ocurren durante el sueño, tales como los terrores nocturnos, el sonambulismo y las pesadillas. Las disomnias son procesos caracterizados por anomalías en la cantidad, calidad o cronología del sueño. Este término incluye trastornos como el insomnio, hipersomnia, narcolepsia, apnea del sueño, entre otros (Sateia, 2014).

El insomnio es el trastorno del sueño más frecuente en la población adulta. Se define como una dificultad persistente en el inicio del sueño, su duración, consolidación o calidad que ocurre a pesar de la existencia de adecuadas circunstancias y oportunidad para el mismo, con repercusión negativa en la calidad de vida y actividad laboral del paciente, incremento de accidentes automovilísticos, entre otras condiciones de riesgo para la salud. La nueva clasificación del insomnio según el DSMV (AAP, 2014), no hace referencia al insomnio primario o secundario, sino que considera al insomnio como una alteración en sí misma, siempre que se cumpla los criterios. Se han descrito tres subtipos fundamentales de insomnio, el crónico, con una duración superior a tres meses, el insomnio de corta duración, inferior a tres meses y otros tipos de insomnio que no cumplen con los criterios temporales de los subtipos anteriores (Sateia, 2014).

La prevalencia del insomnio varía de un 5 a un 50\%, considerando la definición utilizada. Se estima que un tercio de la población adulta sufre de insomnio cuando se consideran los síntomas nocturnos, más esa cifra disminuye para el $10 \%$ cuando se consideran las consecuencias diurnas (Ohayon, 2002). Con el incremento de la edad, aumenta la incidencia de insomnio. En ancianos se produce una disminución de del tiempo total de sueño, con despertares nocturnos frecuentes. Los individuos de mayor edad también presentan enfermedades crónicas con elevada frecuencia y usan fármacos que pueden interferir en la calidad del sueño (FitzGerald et al., 2017). El insomnio es más frecuente en mujeres, con una relación mujer: hombre igual a 3:2. Las variaciones hormonales durante el ciclo menstrual o durante la menopausia pueden afectar la calidad del sueño (Ohayon, 2002).

Las principales causas de insomnio pueden ser divididas en condiciones médicas, psicológicas y ambientales. Las enfermedades cardíacas, respiratorias, endocrinas, digestivas y renales con frecuencia cursan con dificultades para iniciar y mantener un sueño de calidad adecuada. Diversas sustancias que se comportan como estimulantes del sistema nervioso central como la cafeína, alcohol, opioides, inclusive la supresión de alguna de estas sustancias causa insomnio. Algunos fármacos como los descongestionantes nasales, corticosteroides y broncodilatadores también interfieren en la calidad y duración del sueño (Taylor et al., 2007). Entre las enfermedades psiquiátricas que provocan alteraciones del sueño REM y no REM se destacan la depresión mayor, los trastornos de estrés postraumáticos, el desorden de ansiedad generalizada, entre otras (Ohayon \& Roth, 
2003). Las condiciones ambientales influyen decisivamente en la calidad del sueño. Los ambientes con temperaturas extremas, sonidos inadecuados, los turnos de trabajo nocturnos, cambios de latitud que acontecen durante los viajes en avión (Jet lag) son circunstancias asociadas a insomnio y otros trastornos del sueño (Sack et al., 2007).

El insomnio crónico se señala como un fuerte predictor de depresión. Una disminución de las horas de sueño se asocia a incremento de la mortalidad general, principalmente en ancianos. Individuos con insomnio crónico presentan un mayor riesgo de ansiedad, abuso de sustancias nocivas como alcohol, drogas y dependencia de nicotina (Bonnet \& Arand, 2006).

Los trastornos del sueño, principalmente el insomnio crónico se manifiesta con gran frecuencia en las enfermedades psiquiátricas, estando presente dentro de los criterios diagnósticos de la depresión mayor, trastorno bipolar, entre otros. En algunos casos, las alteraciones del patrón de sueño preceden al diagnóstico de la enfermedad psiquiátrica (NIH, 2005). Estudios recientes apuntan la relación entre los trastornos del sueño y la presencia de ideación suicida. La evaluación de la calidad del sueño y la detección de ideación suicida a través de diferentes cuestionarios, permite correlacionar la presencia de trastornos del sueño con la ideación suicida. Entre los cuestionarios más utilizados para evaluar la calidad del sueño tenemos: Sleep Disorders Questionnaire, Cuestionario Oviedo de Calidad del Sueño, Sleep Timing Questionnaire, Wisconsin Sleep Questionnaire, Sleep Disorders Inventary, Pittsburg Sleep Quality Index, entre otros (Lomelí et al., 2008).

El suicidio representa un problema de salud de gran relevancia a nivel mundial, siendo responsable por la pérdida de una vida cada 40 segundos. Diversos datos apuntan que cada año se producen aproximadamente un millón de muertes, de forma global (Bernert et al., 2017). Según la WHO (2014), la conducta suicida se define como un conjunto de actitudes que incluyen la ideación suicida, plan suicida, tentativa de suicidio y el suicidio propiamente dicho.

- Ideación suicida: Pensamientos recurrentes de autoagresión.

- Plan suicida: Proyecto bien estructurado que comprende la forma, el momento y el lugar de cometer el acto suicidio.

- Tentativa de suicídio: Violencia autoprovocada no fatal.

- Suicidio consumado: Acto violento auto provocado en el que se produce la muerte del individuo (McCullumsmith, 2015).

La conducta suicida se asocia a factores sociales, psicológicos, biológicos y ambientales. La identificación de dichos factores de riesgo, así como la intervención oportuna, puede prevenir un desenlace fatal. Entre los factores modificables se encuentran los trastornos del sueño, principalmente cuando las consecuencias de la calidad y la cantidad de las horas de sueño repercuten negativamente en la calidad de vida y el desempeño diurno del sujeto.

Considerar los trastornos del sueño como marcador de ideación suicida, en diferentes contextos clínicos, permite la implementación de estrategias terapéuticas precoces para evitar un probable suicidio (Woznica et al., 2015). La familia, la comunidad y los profesionales de la salud deben prestar todo el apoyo necesario a los pacientes que se encuentren en riesgo de cometer suicidio (Chagas et al., 2020). Desde el punto de vista asistencial, se requiere de una atención interprofesional y centrada en la persona.

La presente revisión sistemática tiene como objetivo analizar artículos que abordaron la relación existente entre los trastornos del sueño y la ideación suicida en diferentes circunstancias, grupos de edades y condiciones patológicas pre-existentes.

\section{Metodologia}

Pregunta de investigación: ¿Los trastornos del ciclo sueño- vigilia pueden ser considerados marcadores de ideación suicida?

El enfoque de este estudio es de carácter cualitativo. Los métodos cualitativos son aquellos en los que la interpretación por parte del investigador con sus opiniones sobre el fenómeno en estudio (Pereira et al., 2018). La metodología de la 
investigación bibliográfica se realiza mediante la revisión periódica o esporádica de un tema específico con el fin de concebir una visión general de un tema determinado (Marconi \& Lakatos, 2011).

Para llevar a cabo la revisión y responder la pregunta de investigación fue realizada una búsqueda en las bases de datos bibliográficos del área de la salud: Medline, Scielo y LILACS durante los meses de mayo a diciembre de 2020. Se incluyeron estudios originales cuantitativos y revisiones cualitativas, así como meta-análisis, publicados en los últimos 10 años en idiomas español, inglés y portugués. Los términos usados para la búsqueda fueron: trastornos del sueño-vigilia, depresión e ideación suicida.

Una vez seleccionados los artículos que cumplían con los criterios de inclusión, se organizaron en las siguientes categorías:

- Trastornos del sueño-vigilia e ideación suicida, abordaje general.

- Trastornos del sueño-vigilia e ideación suicida relacionados con la profesión.

- Trastornos del sueño-vigilia e ideación suicida en pacientes con trastorno depresivo previo.

- Trastornos del sueño-vigilia e ideación suicida en pacientes con trastorno neurológico previo.

- Trastornos del sueño-vigilia e ideación suicida en veteranos de guerra.

- Trastornos del sueño-vigilia e ideación suicida en adolescentes.

- Trastornos del sueño-vigilia e ideación suicida en ancianos.

\section{Resultados}

Después de realizar la búsqueda en las bases de datos consultadas, se encontraron 39 artículos que cumplían con los criterios de selección (Tabla 1).

Tabla 1. Demuestra el número de artículos encontrados en cada base de datos bibliográficos del área de la salud.

\begin{tabular}{|c|c|c|}
\hline Scielo & LILACS & Medline \\
\hline \multicolumn{3}{|c|}{ (Lectura de títulos y resúmenes) } \\
\hline $\begin{array}{l}\text { Relacionados con los trastornos del } \\
\text { sueño y vigilia: } 416 \text { artículos }\end{array}$ & $\begin{array}{l}\text { Relacionados con los trastornos del } \\
\text { sueño y vigilia: } 1205 \text { artículos }\end{array}$ & $\begin{array}{l}\text { Relacionados con los trastornos del } \\
\text { sueño y vigilia: } 3774 \text { artículos }\end{array}$ \\
\hline $\begin{array}{l}\text { Relacionados con ideación suicida: } \\
\qquad 215 \text { artículos }\end{array}$ & $\begin{array}{l}\text { Relacionados con ideación suicida: } \\
\qquad 4067 \text { artículos }\end{array}$ & $\begin{array}{l}\text { Relacionados con ideación suicida: } \\
6342 \text { artículos }\end{array}$ \\
\hline \multicolumn{3}{|c|}{ (Lectura de resúmenes) } \\
\hline & $\begin{array}{l}\text { Relacionados con ambos términos } \\
\text { de búsqueda: } 65 \text { artículos }\end{array}$ & \\
\hline \multicolumn{3}{|c|}{ (Lectura íntegra) } \\
\hline $\begin{array}{l}\text { Cumplen los criterios de selección: } \\
\qquad 3 \text { artículos }\end{array}$ & $\begin{array}{l}\text { Cumplen los criterios de selección: } \\
5 \text { artículos }\end{array}$ & $\begin{array}{l}\text { Cumplen los criterios de selección: } \\
\qquad 36 \text { artículos }\end{array}$ \\
\hline \multicolumn{3}{|c|}{ Artículos repetidos: 5} \\
\hline \multicolumn{3}{|c|}{ Artículos selecionados: 39} \\
\hline
\end{tabular}

Fonte: Autores. 
De los artículos seleccionados, 37 estaban escritos en idioma inglés y 2 en idioma portugués. La base de datos Medline aportó el 79,4\% de los artículos utilizados. Estas publicaciones se organizaron por categorías, para facilitar su análisis, como consta en la Tabla 2 .

Tabla 2. Demuestra el número de artículos en cada categoría.

\begin{tabular}{|c|c|}
\hline Categoría & $\begin{array}{l}\text { ne de } \\
\text { artículos }\end{array}$ \\
\hline $\begin{array}{c}\text { Trastornos del sueño-vigilia e ideación suicida, abordaje } \\
\text { general }\end{array}$ & 6 \\
\hline $\begin{array}{c}\text { Trastornos del suefio-vigilia e ideación suicida } \\
\text { relacionados con la profesión }\end{array}$ & 1 \\
\hline $\begin{array}{c}\text { Trastornos del suefio-vigilia e ideación suicida en } \\
\text { pacientes con trastorno depresivo previo }\end{array}$ & 17 \\
\hline $\begin{array}{l}\text { Trastornos del sueño-vigilia e ideación suicida en } \\
\text { pacientes con trastorno neurológico previo }\end{array}$ & 1 \\
\hline $\begin{array}{c}\text { Trastornos del sueño-vigilia e ideación suicida en } \\
\text { veteranos de guerra }\end{array}$ & 4 \\
\hline $\begin{array}{c}\text { Trastornos del sueño-vigilia e ideación suicida en } \\
\text { adolescentes y jóvenes }\end{array}$ & 8 \\
\hline $\begin{array}{c}\text { Trastornos del sueño-vigilia e ideación suicida en } \\
\text { ancianos }\end{array}$ & 2 \\
\hline Total & 39 \\
\hline
\end{tabular}

Fonte: Autores.

El 87, $1 \%$ de los artículos se corresponden con estudios originales cuantitativos (Tabla 3).

Tabla 3. Demuestra los tipos de estudios analizados.

\begin{tabular}{|c|c|}
\hline Tipos de estudios analizados & $\begin{array}{c}\mathbf{n}^{\circ} \mathrm{de} \\
\text { artículos }\end{array}$ \\
\hline Originales cuantitativos & 34 \\
\hline Revisiones cualitativas & 2 \\
\hline Meta-análisis & 3 \\
\hline Total & 39 \\
\hline
\end{tabular}

Fonte: Autores.

\section{Discusión}

En los últimos años los trastornos del sueño comenzaron a ser considerados como un factor de riesgo modificable en relación con la conducta suicida. Diferentes estudios apuntan que los trastornos del sueño se asocian a incremento del riesgo de ideación suicida, intento suicida y muerte por suicidio (Bae et al., 2013; Bernert et al., 2015; Gelaye et al., 2016; Kim et al., 2013; Li et al., 2013; Nadorff et al., 2011; Pigeon et al., 2012). En concordancia con estos hallazgos, los trastornos del sueño se consideran entre los principales parámetros incluidos en las escalas para evaluar conducta suicida (Abarca et al., 2018).

\section{Trastornos del sueño-vigilia e ideación suicida, abordaje general}

Gelaye y colaboradores aplicaron el Cuestionario de Salud del Paciente-9 (PHQ-9) y el Índice de Calidad del Sueño de Pittsburg (PSQI) a 1054 adultos, de los cuales el 24,3 \% presentó ideación suicida y el 60\% arrojó resultados compatibles con calidad del sueño pobre. Aquellos individuos con calidad de sueño considerada inferior presentaron un incremento de la ideación 
suicida, aproximadamente 3 veces mayor a aquellos que demostraron que su sueño era de calidad adecuada. Concluyen reafirmando la asociación positiva entre pobre calidad del sueño e ideación suicida (Gelaye et al., 2016).

En el 2013 fue publicada la investigación de Kim y colaboradores, realizada en Korea com 15236 participantes mayores de 19 años. Se registró la cantidad de sueño en horas y la presencia de ideación suicida se evaluó considerando la respuesta a la pregunta: ¿Usted ha sentidos deseos de morir durante el último año? Fue posible concluir que la ideación suicida se incrementa cuando las horas diarias de sueño son inferiores a 5 o superiores a 9 (Kim et al., 2013). Resultados similares obtuvieron Bae y colaboradores al aplicar la Escala de depresión del Centro de Estudios Epidemiológicos (CES-D), la Escala de Idiación Suicida de Beck (BSI), el Inventario de la expresión del enojo de Spielberg (STAXI), la Escala de impulsividad de Barret (BSI) a 1000 individuos adultos a los que también se les interrogó en relación a la cantidad de horas de sueño semanales. Los resultados mostraron que la presencia de ideación suicida en la población general se asocia a historia familiar de enfermedad psiquiátrica, humor deprimido, así como duración del sueño nocturno inferior o superior a las horas consideradas como necesarias (Bae et al., 2013).

En Meta-análisis realizado por Li y colaboradores se evaluaron 39 estudios en los que se analizaron 147753 individuos con relación a la presencia de trastornos del sueño y conducta suicida. Se concluye que los trastornos del sueño, particularmente el insomnio y las parasomnias, se asocian al incremento del riesgo relativo de pensamientos suicidas, intento suicida y suicidio consumado (Li et al., 2013). Estos resultados se corresponden con los de Pigeon y colaboradores que encontraron una asociación positiva entre trastornos del sueño y conducta suicida en meta-análisis que estudió a 147753 adultos entre los años 1966 y 2011 (Pigeon et al., 2012).

\section{Trastornos del sueño-vigilia e ideación suicida relacionados con la profesión}

Alves y colaboradores evaluaron una muestra de 53 enfermeros y 111 policías que realizaban turnos de trabajo nocturnos. Em ambos grupos el rango de edades fue de 21 a 30 años. Se les aplicó el Inventario de Ansiedad de Beck y la escala de depresión del Centro de Estudios Epidemiológicos (CES-D) y el cuestionario de Horne-Ostberg. Se evidenció que los enfermeros y los policías militares presentaron un incremento del riesgo de ansiedad y depresión (Alves et al., 2015).

Experimentos animales sugieren que la restricción de sueño crónica puede inducir cambios graduales en estructuras cerebrales muy similares a los que se producen en pacientes deprimidos. De acuerdo con esta hipótesis, la restricción del sueño crónica altera las propiedades fundamentales del sistema neuroendocrino en relación con la respuesta al estrés. Cuando la restricción del sueño se produce de manera crónica, ocurre una disminución de la proliferación celular hipocampal y la neurogénesis, que eventualmente pueden provocar reducción del volumen hipocampal. Estos eventos, unidos a la disminución de la plasticidad hipocampal, contribuyen al desarrollo de trastornos de humor (Palagini et al., 2019).

\section{Trastornos del sueño-vigilia e ideación suicida en pacientes con trastorno depresivo previo}

Los trastornos de humor se encuentran entre los problemas de salud más prevalentes e incapacitantes a nivel mundial. Los trastornos del sueño y particularmente el insomnio son síntomas clínicamente significativos en pacientes con trastorno de humor (Rocha et al., 2016). El insomnio afecta de un 80 a $100 \%$ de los individuos que están pasando por un episodio depresivo y de un 45 a un $55 \%$ de los pacientes con trastorno bipolar en la etapa de transición. Palagini y colaboradores (2019) elaboraron un modelo fisiopatológico que explica la conexión entre los trastornos de humor y el insomnio.

En primer lugar, la disrupción del sueño actúa como un estresor neurobiológico que conlleva a una hiperactivación de las vías del sistema de estrés, influyendo de forma negativa en la neurogénesis hipocampal, plasticidad cerebral y conectividad en la corteza prefrontal y en las regiones cerebrales que regulan el humor, la emoción y la cognición. El insomnio favorece un estado de inflamación crónica con un efecto negativo en la producción de serotonina. También se produce una reducción de los 
factores neurotróficos como el factor neurotrófico cerebral (BDNF), que afecta la plasticidad sináptica y la neurogénesis. Las evidencias apuntan a que los circuitos cerebrales que aparentemente están disregulados cuando existen trastornos del sueño son los mismos que están involucrados en la patogénesis de los trastornos de humor (Palagini et al., 2019). Para Wu y colaboradores la terapia cognitivo comportamental es la estrategia terapéutica no farmacológica más eficaz para mejorar el insomnio y otros parámetros del sueño en pacientes con trastornos depresivos e insomnio (Wu et al., 2015).

Los estudios de Chu y colaboradores sugieren que el insomnio severo está asociado a la presencia de ideación suicida. Evaluar el número de horas de sueño y el grado de socialización de pacientes con trastorno depresivo permite predecir el riesgo de conducta suicida y desarrollar estrategias para evitar un suicidio consumado (Chu et al., 2017). Resultados muy similares fueron obtenidos por los grupos de investigación Bernert et al. (2017), Dell'Osso et al. (2014), Krakow et al. (2011), Lee et al. (2015), McCall et al. (2013), (Nadorff et al., 2014). En cada estudio fueron aplicados diferentes cuestionarios para evaluar la calidad del sueño en pacientes con patologías psiquiátricas previas, siendo evidente la asociación entre parasomnias, disregulación del ritmo circadiano, la severidad de la depresión y la presencia de ideación suicida. Blasco-Fontecilla y su equipo analizó la calidad del sueño en pacientes con depresión mayor, reportando una mayor prevalencia de sueño insuficiente en pacientes con depresión e intento suicida previo, en comparación con aquellos sin historia anterior de intento suicida (BlascoFontecilla et al., 2011).

Shoener y colaboradores examinaron la prevalencia de la coexistencia de los síntomas de insomnio e hipersomnio durante los episodios depresivos y la severidad de la depresión, encontrando una asociación positiva entre dichas variables (Soehner et al., 2014). En el estudio de Selvi et al. (2010) esta asociación fue evidente en pacientes con depresión y en sujetos controles sin diagnóstico previo de depresión. Lai y colaboradores (2014) observaron agregación familiar moderada, mas, significativa, para trastornos del sueño, tiempo de latencia aumentado, disfunción diurna y conducta suicida (Lai et al., 2014).

TRASTORNOS DEL SUEÑO-VIGILIA E IDEACIÓN SUICIDA EN PACIENTES CON TRASTORNO NEUROLÓGICO PREVIO.

Para Wigg y colaboradores los pacientes con epilepsia presentan mayor índice de suicidio y mortalidad global en comparación con la población general. Se reportan factores de riesgo específicos para el suicidio en pacientes epilépticos, como la edad inferior a 50 años, sexo masculino, comienzo temprano de la epilepsia, crisis del lóbulo temporal, presencia de lesiones cerebrales, dificultad para controlar las crisis y la presencia de trastornos del sueño (Wigg et al., 2014).

En la investigación de Wigg participaron 98 pacientes epilépticos que se encontraban en tratamiento estable con antiepilépticos. Fueron aplicados el Inventario de depresión de Beck, el Inventario de ansiedad de Beck, el Inventario de ideación suicida de Beck, el Índice de calidad del sueño de Pittsburg (PSQI) y la Escala de Insomnio de Epworth. La ideación suicida estuvo presente en el 13,3\% de los pacientes, asociada a síntomas de depresión, ansiedad y trastornos del sueño. Se recomienda priorizar y monitorizar los síntomas de depresión, ansiedad y trastornos del sueño en pacientes con epilepsia, principalmente aquellos con epilepsia de difícil control (Wigg et al., 2014).

\section{Trastornos del sueño-vigilia e ideación suicida en veteranos de guerra}

DeBeer y colaboradores estudiaron a 130 veteranos de las guerras de Iraq y Afganistán que presentaron lesiones cerebrales secundarias a trauma y trastornos del sueño. Aplicaron el Índice de calidad del sueño de Pittsburg y la Escala de ideación suicida de Beck en aquellos veteranos de guerra con antecedentes de trauma cerebral, independientemente de la etiología. Se demostró la relación entre injuria cerebral traumática, trastornos del sueño e ideación suicida. El tratamiento farmacológico del insomnio incrementó la probabilidad de acto suicida en este grupo de pacientes (DeBeer et al., 2017).

El grupo de trabajo de Bryan, 2015, analizó la asociación entre la severidad del insomnio con la ideación y el intento suicida en 380 pacientes psiquiátricos que con anterioridad formaron parte de las Fuerzas Aéreas Americanas. Fueron aplicados 
los Inventarios de depresión y de ideación suicida de Beck, El Cuestionario de salud del paciente (PHQ-9) y el Índice de severidad del insomnio. Demostraron que existe asociación entre los trastornos del sueño y la depresión. No fue posible demostrar la relación entre la severidad del insomnio y la ideación suicida (Bryan et al., 2015).

Resultados similares apuntan Chakravorty, 2014 e Trockel, 2015. Chakravorty y colaboradores aplicaron el Cuestionario de Paykel para evaluar la ideación suicida y realizaron una entrevista para evaluar la calidad del sueño a 161 veteranos de guerra con antecedentes de uso de drogas, abuso de alcohol y otros problemas conductuales. La ideación suicida se reportó en $39 \%$ de los veteranos y se asoció a calidad del sueño inferior (Chakravorty et al., 2014). Por su parte, Trockel y su equipo de trabajo demostraron que la terapia cognitivo comportamental mejoró los síntomas depresivos en veteranos de guerra con insomnio y depresión (Trockel et al., 2015).

\section{Trastornos del sueño-vigilia e ideación suicida en adolescentes}

El suicidio es la tercera causa de muerte en el grupo de 15 a 24 años. Entre los adolescentes, los trastornos del sueño están relacionados a ideación suicida y suicidio consumado. La madurez de los procesos cognitivos que se establece durante la adolescencia es un factor clave en la evolución de ideas suicidas hacia el suicidio consumado. Existen diferencias por grupos de edades en relación a la proporción de ideación suicida y suicidio consumado. En menores de 11 años pueden estar presentes las ideas suicidas, mas, raramente se concretizan en un acto suicida. A partir de la pubertad, la tasa de suicidio consumado se incrementa en proporción a la edad, hasta alcanzar la madurez (Wong \& Brower, 2012).

Los trastornos de ansiedad y depresión son causas comunes de insomnio en adolescentes. Otros síntomas asociados a las alteraciones del ciclo sueño-vigilia en adolescentes son: humor irritable, cambios en el peso corporal, agitación psicomotora o retardo psicomotor, fatiga o pérdida de energía, sentimientos de culpa, trastornos de concentración, somnolencia excesiva e incremento del riesgo de accidentes automovilísticos (Koyawala et al., 2015; Sarchiapone et al., 2014).

Los estudios de Wong, 2012, 2016, evidenciaron que en una muestra de 10123 adolescentes que estaban siendo tratados por depresión, abuso de alcohol y drogas ilícitas, la presencia de trastornos del sueño se comportó como un factor predictivo de ideación suicida (Wong \& Brower, 2012; Wong et al., 2016). Resultados muy similares obtuvieron McGlinchey, 2016; Winsler, 2015; Koyawala, 2015; Sarchiapone, 2014 y Kaplan, 2014; quienes realizaran estudios con adolescentes que presentaban disturbios de humor y trastornos del sueño, evidenciando una relación directa entre la calidad del sueño inferior, la reducción de las horas del sueño y la presencia de ideación suicida y tentativa de suicidio (Kaplan et al., 2014; Koyawala et al., 2015; McGlinchey et al., 2017; Sarchiapone et al., 2014; Winsler et al., 2015).

\section{Trastornos del sueño-vigilia e ideación suicida en ancianos}

El proceso de envejecimiento impone varios cambios en el individuo, desde el punto de vista biológico, psicológico y social. Esta situación requiere de una serie de adaptaciones que no siempre ocurren de forma adecuada. Los sentimientos de desesperanza y las ideas suicidas pueden conducir a un suicidio consumado en esta fase de la vida. En dependencia de la situación particular, de cada anciano, este proceso puede ser influenciado por factores externos que actúen como factores protectores, que eviten un intento suicida. En múltiples ocasiones resulta complicado para los profesionales de la salud reconocer a los individuos en riesgo y prevenir el suicidio. Diferentes estudios apuntan como factores de riesgo para desarrollar una conducta suicida a los problemas de salud, incapacidad, dolor crónico, problemas auditivos y visuales, frecuentes en pacientes ancianos (Bishop et al., 2016; Lapierre et al., 2012).

El estudio de Lapierre y colaboradores describe una prevalencia de 5,9\% de ideación suicida en una muestra de 2777 ancianos, estando asociada a enfermedades crónicas y trastonos del sueño (Lapierre et al., 2012). 
En los últimos años algunos estudios evidenciaron una relación entre el consumo de fármacos para controlar el insomnio e incremento de la ideación suicida, particularmente en ancianos. Este grupo de pacientes presenta un riesgo elevado de eventos farmacológicos adversos, mayor vulnerabilidad a sedación excesiva, deterioro cognitivo y delirium. La terapia cognitivocomportamental constituye la primera línea de tratamiento para el insomnio en ancianos (Bishop et al., 2016; Bryan et al., 2015).

\section{Consideraciones Finales}

La presenta revisión sistemática evidenció que los trastornos del sueño son un factor de riesgo independiente en relación con la conducta suicida en diferentes contextos clínicos y entornos socio-culturales. Diversos estudios sustentan la importancia de los trastornos del sueño como factor predictor de conducta suicida, así como la importancia del diagnóstico y tratamiento precoz de los trastornos del sueño.

Finalmente, se cree que los trabajos futuros que discutan este tema son relevantes para el desarrollo de la ciencia. Las investigaciones futuras en esta área desarrollarán nuevas estrategias de prevención del suicidio basadas en el adecuado tratamiento de dichas alteraciones.

\section{Referencias}

AAP. (2014). Manual diagnóstico e estatístico de transtornos mentais DSM-5. (5a ed.). Artmed.

Abarca, C., Gheza, C., Coda, C., \& Elicer, B. (2018). Revisión de la literatura para identificar escalas estandarizadas de evaluación del riesgo suicida en adultos usuarios de la Atención Primaria de Salud. Medwave, 18(5), 7246.

Alves, V. d. M., Santos, M. B. d. F., Nascimento, L. M. S., Ferro, G. C., Silva, L. K. B. d., Tenório, F. E., \& Nardi, A. E. (2015). Suicidal ideation and chronotype assessment in nurses and police officers. MedicalExpress, 2(3), 1-6.

Bae, S.-M., Lee, Y. J., Cho, I. H., Kim, S. J., Im, J. S., \& Cho, S.-J. (2013). Risk factors for suicidal ideation of the general population. J Korean Med Sci, 28(4), 602-607.

Bernert, R. A., Hom, M. A., Iwata, N. G., \& Joiner, T. E. (2017). Objectively Assessed Sleep Variability as an Acute Warning Sign of Suicidal Ideation in a Longitudinal Evaluation of Young Adults at High Suicide Risk. J Clin Psychiatry. 78(6).

Bernert, R. A., Kim, J. S., Iwata, N. G., \& Perlis, M. L. (2015). Sleep disturbances as an evidence-based suicide risk factor. Curr Psychiatry Rep, $17(3)$, 554.

Berry, R. B., Quan, S. F., \& Abreu, A. R. (2020). The AASM Manual for the Scoring of Sleep and Associated Events: Rules, Terminology and Technical Specifications, Version 2.6. American Academy of Sleep Medicine. https://aasm.org/clinical-resources/scoring-manual/

Bishop, T. M., Simons, K. V., King, D. A., \& Pigeon, W. R. (2016). Sleep and Suicide in Older Adults: An Opportunity for Intervention. Sleep and Suicide in Older Adults: An Opportunity for Intervention. Clin Ther, 38(11), 2332-2339.

Blasco-Fontecilla, H., Alegria, A. A., Lopez-Castroman, J., Legido-Gil, T., Saiz-Ruiz, J., Leon, J. d., \& Baca-Garcia, E. (2011). Short self-reported sleep duration and suicidal behavior: a cross-sectional study. J Affect Disord., 133(1-2), 239-246.

Bonnet, M. H., \& Arand, D. L. (2006). Consequences of insomnia. Sleep Med Clin, 1, 351.

Bryan, C. J., Gonzales, J., Rudd, M. D., Bryan, A. O., Clemans, T. A., Ray-Sannerud, B., Wertenberger, E., Leeson, B., Heron, E., Morrow, C. E., \& Etienne, N. (2015). Depression mediates the relation of insomnia severity with suicide risk in three clinical samples of U. S. military personnel. Depress Anxiety, 32(9), $647-655$.

Chagas, J. A. S. d., Matos, T. d. O., Dias, C. A. G. M., Dendasck, C. V., Oliveira, E. d., Araújo, M. H. M. d., \& Fecury, A. A. (2020). Suicide attempts in Macapá, Amapá, Amazon Region, Brazil, in 2018. Revista Científica Multidisciplinar Núcleo do Conhecimento, 3, 17-35. https://www.nucleodoconhecimento.com.br/health/suicide-attempts

Chakravorty, S., Grandner, M. A., Mavandadi, S., Perlis, M. L., Sturgis, E. B., \& Oslin, D. W. (2014). Suicidal ideation in veterans misusing alcohol: relationships with insomnia symptoms and sleep duration. Addict Behav. 39(2), 399-405.

Chu, C., Hom, M. A., Rogers, M. L., Stanley, I. H., Ringer-Moberg, F. B., Podlogar, M. C., Hirsch, J. K., \& Joiner, T. E. (2017). Insomnia and suicide-related behaviors: A multi-study investigation of thwarted belongingness as a distinct explanatory factor. J Affect Disord. 208, 153-162.

DeBeer, B. B., Kimbrel, N. A., Mendoza, C., Davidson, D., Meyer, E. C., Bash, H. L., Gulliver, S. B., \& Morissette, S. B. (2017). Traumatic Brain Injury, Sleep Quality, and Suicidal Ideation in Iraq/Afghanistan Era Veterans. J Nerv Ment Dis. 205(7), 512-516. 201.

Dell'Osso, L., Massimetti, G., Conversano, C., Bertelloni, C. A., Carta, M. G., Ricca, V., \& Carmassi, C. (2014). Alterations in circadian/seasonal rhythms and vegetative functions are related to suicidality in DSM-5 PTSD. BMC Psychiatry, 14, 352. 
FitzGerald, J. M., O'Regan, N., Adamis, D., Timmons, S., Dunne, C. P., Trzepacz, P. T., \& Meagher, D. J. (2017). Sleep-wake cycle disturbances in elderly acute general medical inpatients: longitudinal relationship to delirium and dementia. Alzheimers Dement (Amst), 7, 61-68.

Gelaye, B., Okeiga, J., Ayantoye, I., Berhane, H. Y., Berhane, Y., \& Williams, M. A. (2016). Association of suicidal ideation with poor sleep quality among Ethiopian adults. Sleep Breath, 20(4), 1319-1326.

Kalmbach, D. A., Schneider, L. D., Cheung, J., Bertrand, S. J., Kariharan, T., Pack, A. I., \& Gehrman, P. R. (2017). Genetic basis of chronotype in humans: insights from three landmark gwas. Sleep, 40(2).

Kaplan, S. G., Ali, S. K., Simpson, B., Britt, V., \& McCall, W. V. (2014). Associations between sleep disturbance and suicidal ideation in adolescents admitted to an inpatient psychiatric unit. Int J Adolesc Med Health, 26(3), 411-416.

Kim, J.-H., Park, E.-C., Cho, W.-H., Park, C. Y., Choi, W.-J., \& Chang, H.-S. (2013). Association between total sleep duration and suicidal ideation among the Korean general adult population. Sleep, 36(10), 1563-1572

Koyawala, N., Stevens, J., McBee-Strayer, S. M., Cannon, E. A., \& Bridge, J. A. (2015). Sleep problems and suicide attempts among adolescents: a case-control study. Behav Sleep Med., 13(4), 285-295.

Krakow, B., Ribeiro, J. D., Ulibarri, V. A., Krakow, J., \& Jr., T. E. J. (2011). Sleep disturbances and suicidal ideation in sleep medical center patients. $J$ Affect Disord, 131(1-3), 422-427.

Lai, Y.-C., Huang, M.-C., Chen, H.-C., Lu, M.-K., Chiu, Y.-H., Shen, W. W., Lu, R.-B., \& Kuo, P.-H. (2014). Familiality and clinical outcomes of sleep disturbances in major depressive and bipolar disorders. J Psychosom Res. 76(1), 61-67.

Lapierre, S., Boyer, R., Desjardins, S., Dubé, M., Lorrain, D., Préville, M., \& Brassard, J. (2012). Daily hassles, physical illness, and sleep problems in older adults with wishes to die. Int Psychogeriatr., 24(2), 243-252.

Lee, M.-S., Shin, J.-S., Lee, J., Lee, Y. J., Kim, M.-r., Park, K. B., Shin, D., Cho, J.-H., \& Ha, I.-H. (2015). The association between mental health, chronic disease and sleep duration in Koreans: a cross-sectional study. BMC Public Health, 15, 1200.

Li, S. X., Lam, S. P., \& Wing, Y. K. (2013). Looking beyond the sleep complaints: a link to suicidality. J Clin Psychiatry, 74(2), 192.

Lomelí, H. A., Pérez-Olmos, I., Talero-Gutiérrez, C., Moreno, C. B., González-Reyes, R., Palacios, L., Peña, F. d. 1., \& Muñoz-Delgado, J. (2008). Escalas y cuestionarios para evaluar el sueño: una revisión. Actas Esp Psiquiatr, 36(1), 50-59.

Marconi, M. A., \& Lakatos, E. M. (2011). Metodologia do trabalho científico: procedimentos básicos, pesquisa bibliográfica, projeto e relatório, publicações e trabalhos cientificos. Atlas.

McCall, W. V., Batson, N., Webster, M., Case, L. D., Joshi, I., Derreberry, T., McDonough, A., \& Farris, S. R. (2013). Nightmares and dysfunctional beliefs about sleep mediate the effect of insomnia symptoms on suicidal ideation. J Clin Sleep Med., 9(2), 135-140.

McCullumsmith, C. (2015). Laying the groundwork for standardized assessment of suicidal behavior. J Clin Psychiatry, 76(10), e1333-1335.

McGlinchey, E. L., Courtney-Seidler, E. A., German, M., \& Miller, A. L. (2017). The Role of Sleep Disturbance in Suicidal and Nonsuicidal Self-Injurious Behavior among Adolescents. Suicide Life Threat Behav, 47(1), 103-111.

Nadorff, M. R., Ellis, T. E., Allen, J. G., Winer, E. S., \& Herrera, S. (2014). Presence and persistence of sleep-related symptoms and suicidal ideation in psychiatric inpatients. Crisis, 35(6), 398-405.

Nadorff, M. R., Nazem, S., \& Fiske, A. (2011). Insomnia symptoms, nightmares, and suicidal ideation in a college student sample. Sleep, 34(1), 93-98.

NIH. (2005). National Institutes of Health State of the Science Conference statement on Manifestations and Management of Chronic Insomnia in Adults, June 13-15, 2005. Sleep, 28(9), 1049-1045.

Ohayon, M. M. (2002). Epidemiology of insomnia: what we know and what we still need to learn. Sleep Med Rev., 6(2), 97-111.

Ohayon, M. M., \& Roth, T. (2003). Place of chronic insomnia in the course of depressive and anxiety disorders. J Psychiatr Res., 37(1), 9-15.

Palagini, L., Bastien, C. H., Marazziti, D., Ellis, J. G., \& Riemann, D. (2019). The key role of insomnia and sleep loss in the dysregulation of multiple systems involved in mood disorders: A proposed model. J Sleep Res., 28(6), e12841.

Pereira, AS, Shitsuka, DM, Parreira, FJ \& Shitsuka, R. (2018). Metodologia da Pesquisa Científica. UFSM.

Pigeon, W. R., Pinquart, M., \& Conner, K. (2012). Meta-analysis of sleep disturbance and suicidal thoughts and behaviors. J Clin Psychiatry, 73(9), 160-167.

Rocha, P. M., Neves, F. S., \& Corrêa, H. (2016). Sleep quality and suicidal behavior in euthymic bipolar patients. Trends Psychiatry Psychother, 38(3), 183184.

Sack, R. L., Auckley, D., Auger, R. R., Carskadon, M. A., Jr, K. P. W., Vitiello, M. V., \& Zhdanova, I. V. (2007). Circadian rhythm sleep disorders: part I, basic principles, shift work and jet lag disorders. An American Academy of Sleep Medicine review. Sleep, 30(11), 1460-1483.

Sarchiapone, M., Mandelli, L., Carli, V., Iosue, M., Wasserman, C., Hadlaczky, G., Hoven, C., Apter, A., Balázs, J., Bobes, J., Brunner, R., Corcoran, P., Cosman, D., Haring, C., Kaess, M., Keeley, H., Keresztény, A., Kahn, J., Poštuvan, V., Mars, U., Sáiz, P., Varnik, P., Sisask, M., \& Wasserman, D. (2014). Hours of sleep in adolescents and its association with anxiety, emotional concerns, and suicidal ideation. Sleep Medicine, 15(2), $248-254$.

Sateia, M. J. (2014). International Classification of Sleep Disorders-Third Edition. Contemporary Reviews in Sleep Medicine, 146(5), 1387-1394. 
Research, Society and Development, v. 10, n. 2, e34610212736, 2021

(CC BY 4.0) | ISSN 2525-3409 | DOI: http://dx.doi.org/10.33448/rsd-v10i2.12736

Selvi, Y., Aydın, A., Boysan, M., Atli, A., Agargun, M., \& Besiroglu, L. (2010). Associations between chronotype, sleep quality, suicidality, and depressive symptoms in patients with major depression and healthy controls. Chronobiol Int., 27(9-10), 1813-1828.

Soehner, A. M., Kaplan, K. A., \& Harvey, A. G. (2014). Prevalence and clinical correlates of co-occurring insomnia and hypersomnia symptoms in depression. J Affect Disord, 167, 93-97.

Takahashi, J. S. (2017). Transcriptional architecture of the mammalian circadian clock. Nat Rev Genet., 8, V164-179.

Taylor, D. J., J., M. L., Lichstein, K. L., Durrence, H. H., W., R. B., \& J., B. A. (2007). Comorbidity of chronic insomnia with medical problems. Sleep, 30(2), 213-218.

Trockel, M., Karlin, B. E., Taylor, C. B., Brown, G. K., \& Manber, R. (2015). Effects of cognitive behavioral therapy for insomnia on suicidal ideation in veterans. Sleep, 38(2), 259-265.

WHO. (2014). Preventing suicide: a global imperative. World Health Organization. https://apps.who.int/iris/bitstream/handle/10665/131056/9789 241564779_eng.pdf;jsessionid=58DDC8CEB4C749C25073F32B9D36A243? sequence=1

Wigg, C. M., Filgueiro, A., \& Gomes, M. (2014). A relação entre qualidade do sono, depressão e ansiedade em pacientes com epilepsia e ideação suicida. Arq Neuropsiquiatr, 72(5), 344-348.

Winsler, A., Deutsch, A., Vorona, R. D., Payne, P. A., \& Szklo-Coxe, M. (2015). Sleepless in Fairfax: the difference one more hour of sleep can make for teen hopelessness, suicidal ideation, and substance use. J Youth Adolesc, 44(2), 362-378.

Wong, M. M., \& Brower, K. J. (2012). The prospective relationship between sleep problems and suicidal behavior in the National Longitudinal Study of Adolescent Health. J Psychiatr Res., 46(7), 953-959.

Wong, M. M., Brower, K. J., \& Craun, E. A. (2016). Insomnia symptoms and suicidality in the National Comorbidity Survey - Adolescent Supplement. $J$ Psychiatr Res., 81, 1-8.

Woznica, A. A., Carney, C. E., Kuo, J. R., \& Moss, T. G. (2015). The insomnia and suicide link: toward an enhanced understand ing of this relationship. Sleep Med Rev., 22, 37-46.

Wu, J. Q., Appleman, E. R., \& Salazar, R. D. (2015). Cognitive Behavioral Therapy for Insomnia Comorbid with Psychiatric and Medical Conditions: A Metaanalysis. JAMA Intern Med., 175(9), 1461-1472. 